\title{
Integrated optomechanical circuits
}

\author{
J. Roels ${ }^{*}$, B. Maes, R. Baets, D. Van Thourhout \\ Photonics Research Group, INTEC Department, Ghent University-imec, B-9000 Gent, Belgium \\ *E-mail: Joris.Roels@intec.ugent.be,
}

\begin{abstract}
Optomechanical circuits are a promising candidate to realize various signal processing functions on a chip. In this paper we review several different NOMS structures fabricated in a silicon-on-insulator platform.

(C) 2010 Optical society of America
\end{abstract}

OCIS-codes: (130.3120) Integrated optics devices; (250.4745) Optomechanics

\section{Introduction}

Over the past two decades a remarkable progress has been made in controlling and trapping microparticles in a laser beam waist [1] using the strong field gradients that are present in this free space laser beam. Also in microphotonic devices strong field gradients can be encountered [2], so not surprisingly it was only a matter of time till Micro- (and Nano) OptoMechanical Systems (MOMS \& NOMS) have been proposed as alternative for classical Micro-OptoElectro-Mechanical Systems (MOEMS) [3]. The convenience of NOMS lies in the fact that the driving force is provided by the light itself and no additional electrical driving circuitry is needed. Therefore fabrication complexity is reduced (less fabrication steps, less chip area consumed). In addition also some other issues that might comprise the device functionality can be avoided (e.g. transmission line effects for high frequencies, possible reduction of the mechanical Q-factor through the contacting). All-optical switching functionality via a NOMS circuit has already been demonstrated [4]. On the more fundamental level the recent developments in optomechanics are quickly leading towards intriguing physical phenomena such as observation of quantum mechanical behavior of a micromechanical oscillator [5][6]. However when considering practical applications (e.g. on chip reference oscillators and widely tunable RF-filters in the optical domain) on the shorter term the need for fully integrated devices (both access waveguides and optomechanical components on a chip) becomes more stringent.

In this paper, we will give an overview of a variety of integrated optomechanical devices on a silicon-on-insulator platform.

\section{Forces between parallel waveguides}

Using a silicon-on-insulator material (SOI) platform with its high refractive index contrast, it is possible to make very compact nanophotonic circuits. By applying Deep-Ultra-Violet lithography and subsequent RIE etching [7] it is even possible to do this on a large (potentially inexpensive) scale which might be an additional asset when considering practical applications. After the definition of the passive nanophotonic circuit typically a part of a waveguide is made freestanding by underetching. This is done by applying a resist mask and etching with buffered HF during an appropriate time. Light can be coupled in and out of the chip using two grating couplers. The measurement is performed in a vacuum environment $(\mathrm{p}<<1 \mathrm{mBar}$ ). This way air damping is avoided and the mechanical quality factor of the micromechanical resonators under study is enhanced significantly. An example of an optomechanical component is shown in Fig. 1a where two parallel waveguides are underetched. In this system typically two guided modes are present. While the ground mode is providing a gap closing force, the first order mode pushes the waveguides apart. In the simplest excitation scheme both modes are excited equally which results in a net in plane attractive force [8]. In an alternative configuration a single waveguide can be attracted to the substrate, resulting in an out of plane attractive force [9]. When implementing an asymmetric Mach-Zehnder in front of the coupler the modes can be controlled by sweeping the wavelength. By consequence both attractive and 
repulsive forces can be obtained (Fig.1b) [10][11]. The induced vibration changes the gap between the waveguides periodically and by consequence the coupling between the waveguides is modulated. Using a probe laser the varying coupling is translated in a weakly power modulated output probe signal which is detected with an electrical spectrum analyzer.

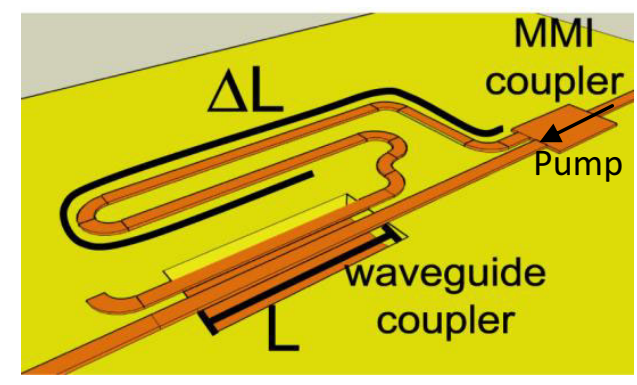

(a)

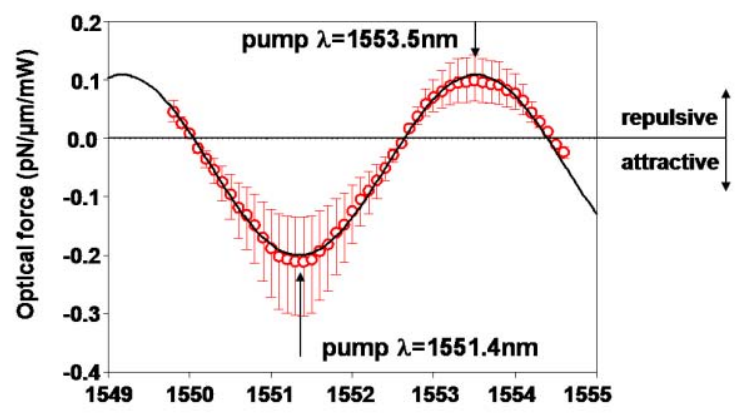

(b)

Fig. 1. (a) Schematic structure of the structure used in [10] to select between the two propagating modes. (b) The optical force is varying from attractive to repulsive in function of the wavelength.

\section{Slotted waveguide}

In a slotted waveguide the light is mainly confined in the air gap as can be seen in Fig. 2a. Due to the small gap size and the related strong field gradients slotted waveguides exhibit relatively large optomechanical effects (the optomechanical parameter $\mathrm{g}_{\mathrm{OM}}=\mathrm{d} \omega / \mathrm{dg}$ in theory can reach values up to $100 \mathrm{GHz} / \mathrm{nm}$ ) and larger forces compared to the parallel waveguide case can be predicted (Fig. 2b). In Fig. 2c an experimental vibration spectrum is shown [12] from a $12 \mu \mathrm{m}$ long freestanding slotted waveguide (beam width $230 \mathrm{~nm}$ and height $=220 \mathrm{~nm}$, gap $=120 \mathrm{~nm}$ ). The vibration spectrum shows a peak of approximately $2.5 \mathrm{~nm}$. The jump is caused by the large vibration amplitude that enables the inset of non-linear mechanical spring hardening. The slot waveguide was placed in a Fabry-Pérot cavity to translate the vibration induced phase modulation into power modulation. The relatively high propagation losses (estimated at $10 \mathrm{db} / \mathrm{cm}$ ) are currently limiting exploitation of the slot in a high finesse optical resonator.

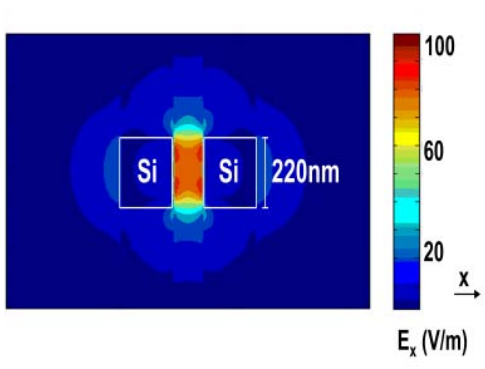

(a)

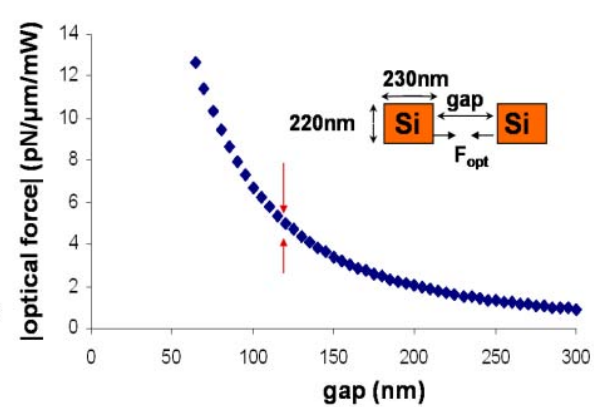

(b)

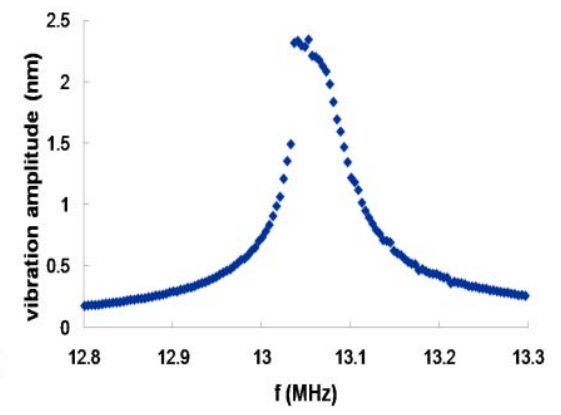

(c)

Fig. 2. (a) Slotted waveguide field profile.- (b) Simulation of the optical force.- (c) Vibration amplitude spectrum for $1 \mathrm{~mW}$ modulated optical power, the spectrum shows a jump non-linearity due to mechanical spring hardening.

\section{Racetrack resonator}

The micromechanical oscillator can also be built in an optical ring or racetrack resonator. For this purpose a part of the racetrack resonator is made freestanding. If the created gap towards the substrate is not too large, again out of plane optomechanical interaction is possible between the waveguide and the $\mathrm{SiO}_{2}$ substrate [13], however it is enhanced by the optical resonator (finesse $\approx 200$ ). The brownian displacement noise of the ground mode of such a 


\section{IMF4.pdf}

freestanding beam is shown in Fig. 3a with resonance frequency around $105.55 \mathrm{MHz}$ and the mechanical Q-factor around 370. In Figure $3 \mathrm{~b}$ the dip in the transmitted power (measured at the detector) is shown when the light in the racetrack is on resonance. The jump in the transmission spectrum is caused by a thermal bistability that origins from two-photon-absorption in the silicon.

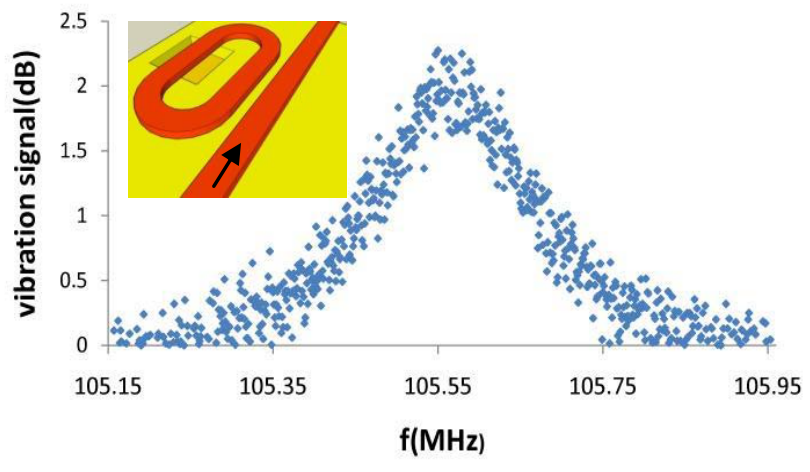

(a)

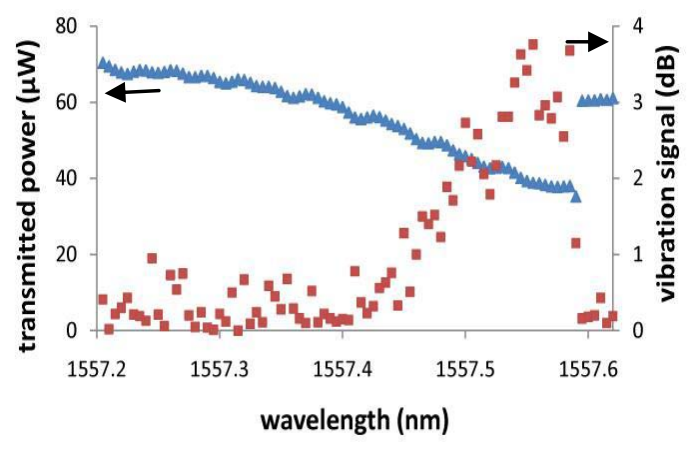

(b)

Fig. 3. (a) thermal 'brownian' response of a freestanding beam in a racetrack resonator for $\lambda=1557.5$; schematic representation of the structure (inset) (b) on resonance transmission dip (triangles) with sawtooth profile and the corresponding vibration signal (squares) (normalized for noise floor, $0 \mathrm{~dB}=$ no signal)

When light is circulating inside the resonator we can pick up the signal of the fundamental mode $(105.55 \mathrm{MHz})$ of the released beam (length $2.6 \mu \mathrm{m}$, width $500 \mathrm{~nm}$, thickness $=220 \mathrm{~nm}$ ) resulting in a sensitivity of $8 \mathrm{fm} / \mathrm{rtHz}$. This sensitivity is clearly limited by the two-photon-absorption effect which induces extra losses and broadens the resonance peak. Slotted waveguides or simply larger wavelengths might reduce the two photon absorption effect.

\section{Conclusion}

We have demonstrated repulsive and attractive forces between waveguides. Slotted waveguides and racetrack resonators are possible candidates to enhance optomechanical effects towards fully integrated NOMS circuits.

\section{Acknowledgement}

This work is supported by the IWT-SBO project "Gemini" and the IAP-project "Photonics@be". B. Maes acknowledges the Fund for Scientific research Flanders for a post-doctoral grant.

\section{References}

1. S. Chu, "Laser manipulation of atoms and particles", Science 253, 861-866 (1991).

2. D. Van Thourhout and J. Roels, "Optomechanical device actuation through the optical gradient force", Nature Photonics 4, 211-217 (2010).

3. M.L. Povinelli, et al, "Evanescent-wave bonding between optical waveguides", Optics Letters, 30, 3042-3044 (2005).

4. J. Rosenberg, Q.Lin, O. Painter, "Static and dynamic wavelength routing via the gradient optical force", Nature Photonics 3, 478-483 (2009)

5. T.J. Kippenberg and K. Vahala, "Cavity optomechanics: Back-action at the mesoscale", Science 321, 1172-1176 (2008)

6. M. Notomi, H. Taniyama, S. Mitsugi and E. Kuramochi "Optomechanical wavelength and energy conversion in high-Q double-layer cavities of photonic crystal slabs. Phys. Rev Lett. 97, 023903 (2006)

7. S.K. Selvaraja, et al., "Fabrication of photonic wire and crystal circuits in silicon-on-insulator using $193 \mathrm{~nm}$ optical lithography", Journal of Lightwave Technology 27, 4706-4083 (2009)

8. J. Roels, et al, "Optomechanical interactions between nanophotonic wires on a silicon-on-insulator chip ", Optical MEMS \& Nanophotonics 2009, United States, p.33-34 (2009)

9. M. Li, W. Pernice and H.X. Tang, "Harnessing optical forces in integrated photonic circuits", Nature 456, 480-484 (2008)

10. J. Roels, et al., "Tunable optical forces between nanophotonic wires", Nature Nanotechnology 4, 510-513 (2009)

11. M. Li, W. Pernice and H.X. Tang, "Tunable bipolar interactions between guided lightwaves", Nature Photonics 3, 464-468 (2009)

12. J. Roels, B. Maes, D. Van Thourhout and R. Baets, "Optical gradient force in a slot waveguide on a Silicon-on-insulator-chip", $22^{\text {nd }}$ Annual Meeting of the IEEE Photonics Society, Turkey, p.223-224 (2009)

13. W.H.P. Pernice, M. Li and H.X. Tang, "Optomechanical coupling in photonic crystal supported nanomechanical waveguides, Optics Express 17,12424-12432 (2009) 